\title{
LabVIEW Based Determination of Oxygen Saturation from ECG Signals
}

\author{
G.Nalinashini, N.Padmavathi, M.Aravindan
}

\begin{abstract}
This research paper demonstrates the measurement of oxygen saturation (SAO2) from the ECG signal using LabVIEW 11.0 .SAO2 is the percentage of hemoglobin molecules that contain oxygen. The optimum level of SAO2 in a human being should be 92\%. When it goes below $90 \%$ it causes hypoxemia (anemia). When SAO2 reduces below 65\%, it will cause damage in the functioning of mental activity. When it reduces below 55\% it causes unconsciousness. Hence sustaining the optimum value of $\mathrm{SAO2}$ is crucial. Hence maintaining optimum level of SAO2 is important. The latest technology which utilizes pulse oximeter has its own drawbacks. To overcome these disadvantages, a new methodology by which $\mathrm{SAO2}$ can be measured from ECG signals is proposed. The ECG signal from the patient is acquired by NI USB 6211 DAQ module. By determining the pulse numbers from the ECG waveform, the SAO2 level can be determined by suitable mathematical computations. Diagnostics and implementing control action can be done with the support of LabVIEW software.
\end{abstract}

Keywords : SAO2, ECG signal, LabVIEW 11.0, NI USB 6211.

\section{INTRODUCTION}

ECG being the important electric potential of our body. Any heart abnormalities and disfunctioning can be diagnosed using this ECG $[1,2]$ To do this task different types of oximeters are used. But still it suffers from noise characteristics and bias inaccuracies [4]. Several manufacturers designed pulse oximeters to determine $\% \mathrm{SaO} 2$ values [5] \& [6]. Pulse oximetry has become the standard clinical method for monitoring patient's blood oxygen saturation and heart rate. Therefore hospitals need a straightforward means to check the accuracy of pulse oximeters. Commercial pulse oximeter testers often cannot be afforded since they are costly [7].

This project work demonstrates that ECGs are used to not only

determine the parameters of cardiac system but also the respiratory parameter. A new methodology is proposed to determine SAO2 from ECG signal. SAO2 is the short form for blood oxygen saturation. The percentage of hemoglobin molecules that contain oxygen can be measured.If the value goes down, then it means that the blood holds insufficient oxygen to properly oxygenate tissues. It normally depends on

Revised Manuscript Received on November 13, 2019.

* Correspondence Author

G.Nalinashini, EIE, RMD Engineering College,Chennai, Email:gns.eie@rmd.ac.in

N.Padmavathi, EIE, RMD Engineering College,Chennai, Email:npi.eie@rmd.ac.in

M.Aravindan, EIE, RMD Engineering College,Chennai,

Email:jenefaravind@gmail.com the person and the problem. Most people have SAO2 $>92 \%$. A level of SAO2 below $88 \%$ is not normal .To obtain the $\mathrm{SAO} 2$ in arterial blood from ECG signal, we have established a relationship between heart rate and $\mathrm{SAO} 2$ with the help of applied mathematics. A standard ECG signal is shown in the Figure 1.

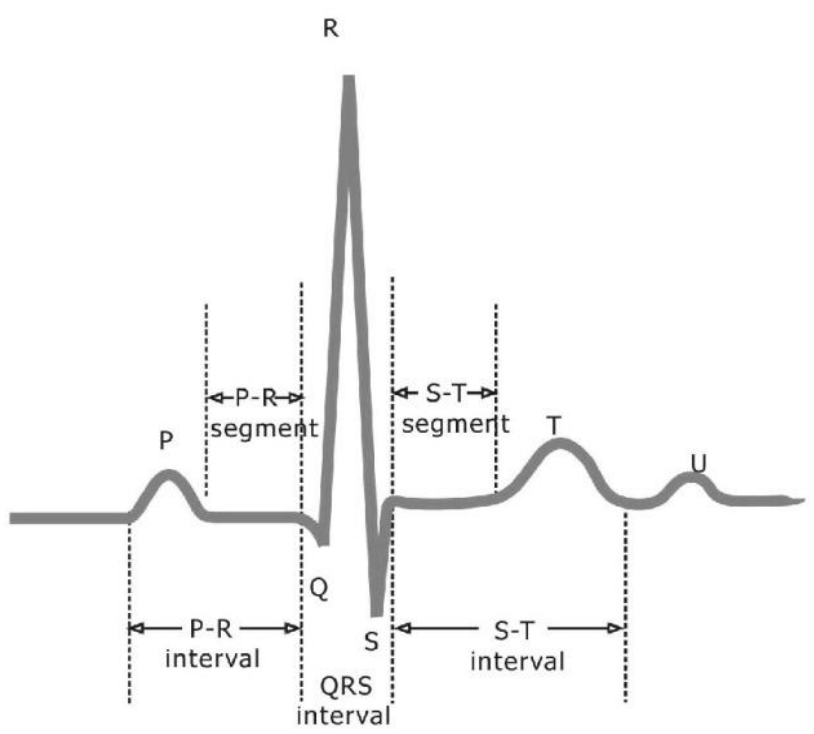

Fig.1 Typical ECG Signal.

\section{DETERMINATION OF SAO2}

Important Terminologies

1) Heart Rate( HR): Number of times our heart beats per minute. The normal heart rate of a normal healthy adult person/human is around 70-72 beats/min.

Cardiac Output (CO): The amount of blood the heart delivers through the circulatory system in a minute. If the average heart rate is 70-72 per minute then cardiac output is $5 \mathrm{Lpm}$. This will increase during the workouts.

2) Stroke volume (SV): The amount of blood delivered by every shrinkage of the ventricles is the stroke volume. Stroke volume is increased when the escalated blood supply is required to meet requirements of the sufficient oxygen and nutrients.

3) SAO2: Oxygen saturation is the ratio of cumulative effects of concentration of oxyhemoglobin and concentration of haemoglobin to the concentration of oxyhaemoglobin 
2.2. Mathematical Calculations

$$
\begin{aligned}
& \mathrm{VO} 2=\mathrm{SV} * \mathrm{HR} \\
& \mathrm{CO}=\mathrm{SV} * \mathrm{HR}
\end{aligned}
$$

Where

$S V \rightarrow$ Stroke Volume in $\mathrm{ml}$

$H R \rightarrow$ Heart Rate in beats/min

$P R \rightarrow$ Pulse Rate in beats/min

$\mathrm{CO} \rightarrow$ Cardiac Output in lit/minute

Comparing equation [1] \& \{2], we get

Taking the $\mathrm{HR}$ as 72 beats/min as a standard value, the value of $\mathrm{CO}$ will be 5 lit $/ \mathrm{min}$.

So for ' $\mathrm{X}$ ' pulses (condition during heart irregularity) we can determine the value of $\mathrm{CO}$ as,

$$
\begin{aligned}
& V O 2=X *(5 \div 72) \\
& \mathrm{CO}=\mathrm{X} *(5 \div 72)
\end{aligned}
$$

Similarly, Considering the normal HR as 72 beats/min the $\mathrm{SAO} 2$ is $100 \%$. So for ' $\mathrm{X}$ ' heart beats (diseased condition) we can determine the $\mathrm{SAO} 2$ by

$$
\mathrm{SAO} 2 \Rightarrow \mathrm{X} *(100 \div 72)
$$

The above equation can still be made more accurate. If a surgery is anticipated for any patient, then the heart rate (HR) and oxygen saturation ( $\mathrm{SAO} 2_{\text {normal }}$ has to be measured. Hence Equation [6] can be modified as shown below.

$$
\mathrm{SAO} 2 \Rightarrow \mathrm{X} *(\mathrm{SAO} 2 \text { normal } \div \mathrm{HR})
$$

Hence SAO2 can be studied using Equation [7]

\section{LabVIEW based determination of $\mathrm{SAO} 2$}

The basic block diagram representing acquisition of ECG signal is given in Figure 2

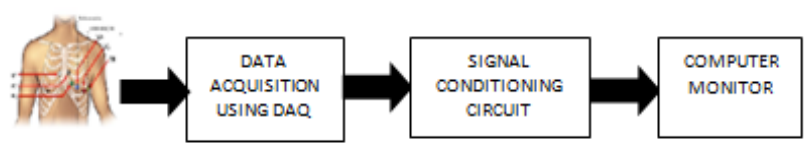

Fig.2 Acquisition of ECG Signal

3.1 Electrodes:

Various types of "ECG electrodes" are available in market, but the most simplest and common one is Surface electrodes which is been used in this project. The electrodes will sense the bio potentials from the body. These signals are amplified by the amplifier so that the signals are measureable

3.2 Data Acquisition Module:

The National Instruments USB-6211 is a multifunction data acquisition (DAQ) module which is bus powered and with high sampling rates. It handless 16 analog inputs; 250 $\mathrm{kS} / \mathrm{s}$ single-channel sampling rate; two analog outputs; four

$$
\mathrm{VO} 2 \Rightarrow \mathrm{CO}
$$

digital input lines; four digital output lines; four programmable input ranges $( \pm 0.2$ to $\pm 10 \mathrm{~V})$ per channel; digital triggering; and two counter/timers. The NI USB-6211 is used for mobile applications. Plug-and-play installation reduces the installation and configuration time. Connection through direct screw-terminal reduces the csts and simplifies connections of the signal. This product does not require external power

\subsection{LabVIEW:}

LabVIEW (short for Laboratory Virtual Instrument Engineering Workbench) is a system-design platform and development environment for a visual programming language from National Instruments. The acquired ECG signal is fed into the system which supports LabVIEW 11.0 software to proceed with the display of the signal and further mathematical calculations. NI-DAQmx driver is installed. It provides measurement services software. The signal processing is done using Biomedical tool kit and Advanced signal processing toolkit.

\subsection{Block Diagram:}

The ECG signals are simulated by using the LabVIEW measurement file. One - dimensional data can be expressed with the LabVIEW Measurement (.lvm) format .The unfiltered ECG signals are filtered using the Notch filter, Bandpass filter and Wavelet denoise Express VI's. The Heart rate is obtained by further calculations and transformations. The Oxygen saturation is calculated using the equation [6]. The assumed values of normal Heart rate and Oxygen saturation is substituted in Equation [7] to measure the SAO2 value. The Block diagram is shown below

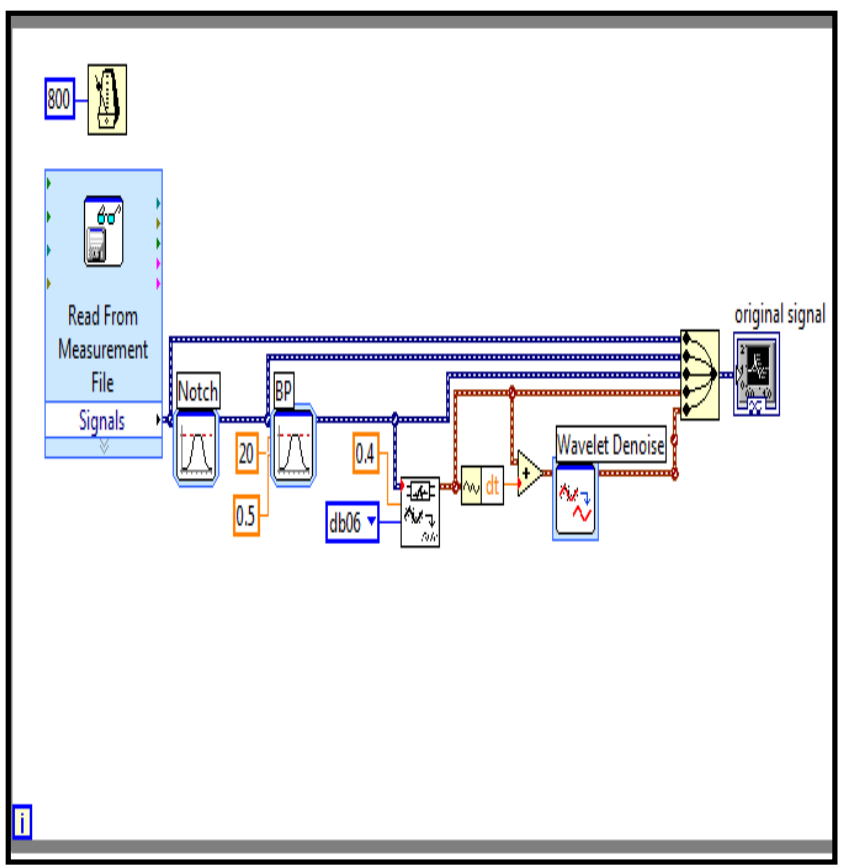

Fig 3. Reading ECG Signal data from the measurement file 


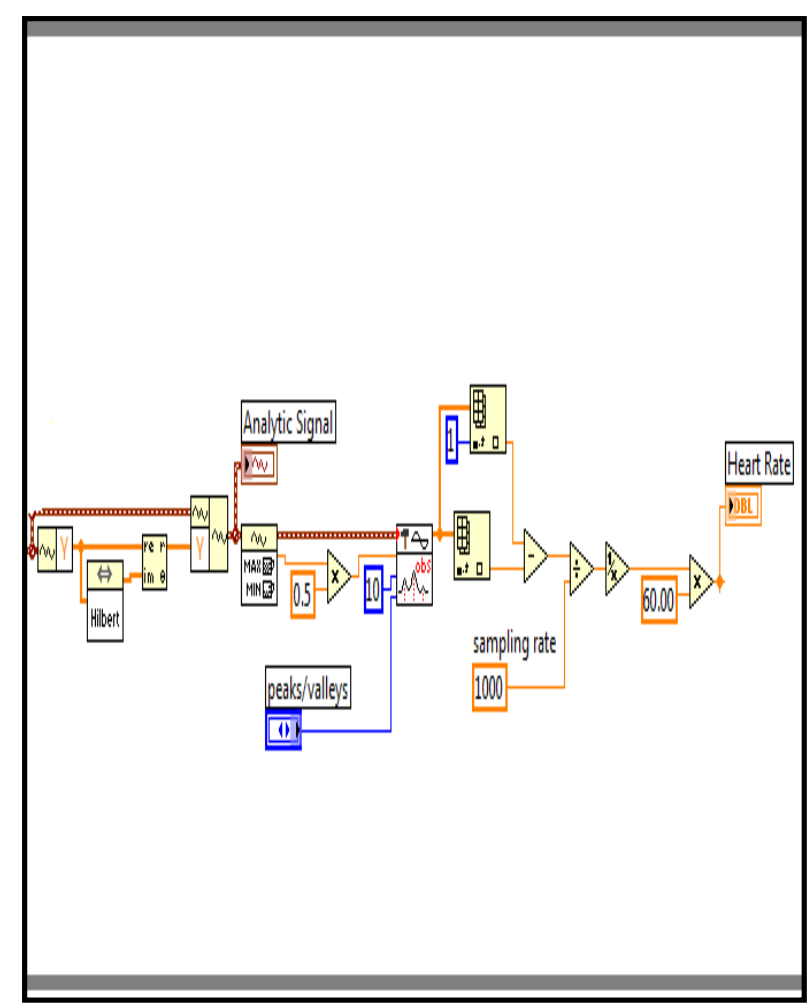

Fig4. Determination of Heart rate

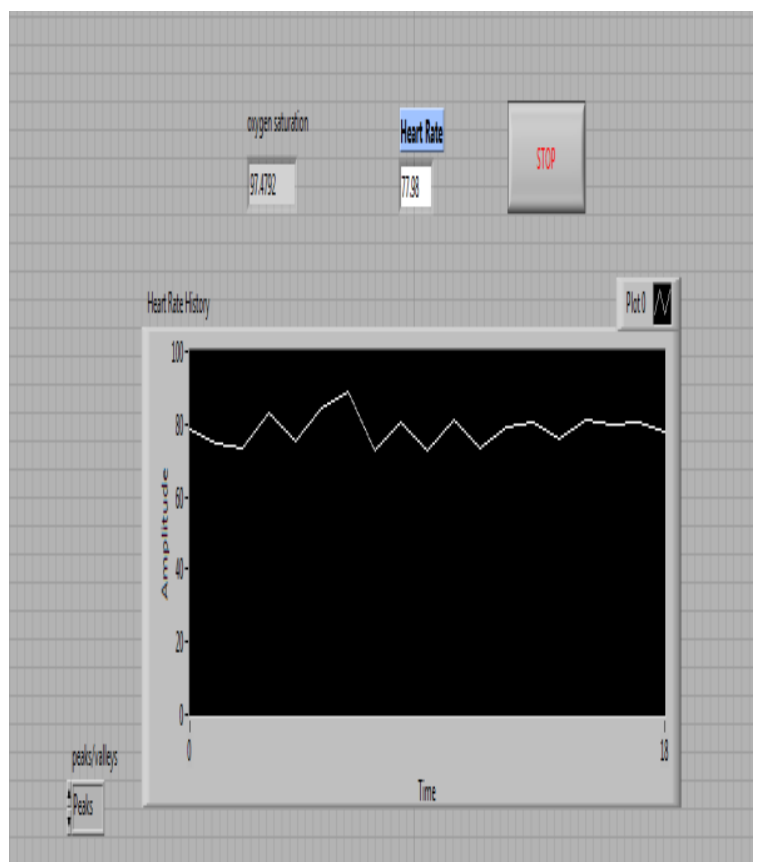

Fig 4.Front panel of VI showing the values of Heart rate and $\mathrm{SAO} 2$
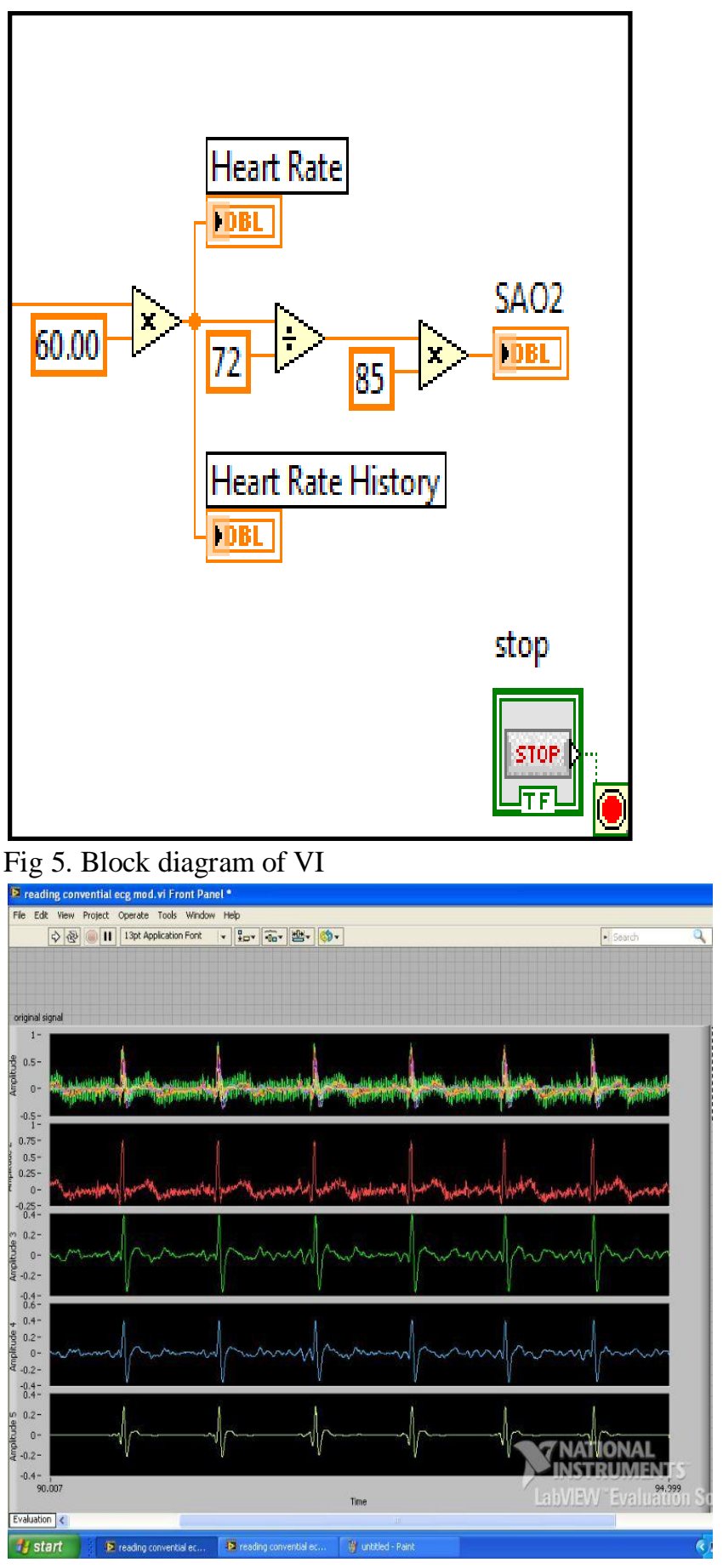

Fig 6 Front panel of VI showing ECG signal

The Front panel shows the filtered ECG signals. Every stage of filtering is shown in the merged graph. The continuous numeric display of the Heart beat rate per minute and Oxygen Saturation is also shown.

\section{CONCLUSION}

Thus a detailed process of LabVIEW based acquisition of ECG signal and measuring the amount of SAO2 from ECG has been discussed in this paper. Apart from determining SAO2, Heart rate, Cardiac Output, Volume of oxygen uptake and saturation pressure of oxygen can be determined 


\section{REFERENCES}

1. Handbook of Biomedical Instrumentation by R.S.Khandpur

2. Biomedical Instrumentation by Marvin.D.Weiss

3. Medical Electronics and Biomedical Instrumentation by C. S. Raja Rao, Sujoy K. Guha

4. Acharya, S.; Rajasekar, A.; Shender, B.S.; Hrebien, L.; Kam, M., "Pulse oximeter signal modeling and fusion for hypoxia monitoring," Information Fusion (FUSION), 2014 17th International Conference on , vol., no., pp.1,8, 7-10 July 2014

5. O'Reilly, G.O.; Tuohy, B., "Methods of assessment of pulse oximeters," Pulse Oximetry: A Critical Appraisal, IEE Colloquium on , vol., no., pp.6/1,6/7.

6. Biology Society, 1988. Proceedings of the Annual International Conference of the IEEE, vol., no., pp.1779,1780 vol.4.

7. Deni, H.; Muratore, D.M.; Malkin, R.A., "Development of a pulse oximeter analyzer for the developing world," Bioengineering Conference, 2005. Proceedings of the IEEE 31st Annual Northeast , vol., no., pp.227,228, 2-3 April 2005 http://www.wseas.us/e-library/conferences/2007athensel/papers/561-3 12.pdf

8. http://lib.bioinfo.pl/paper:16428349

9. https://sites.google.com/site/mbabtechprojects/report-on-spo2-measur ement-using-ecg

10. [11]http://www.ligo.caltech.edu/docs/T/T050162-00.pdf.

\section{AUTHORS PROFILE}

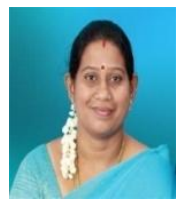

Dr.G.Nalinashini working as Associate Professor in the department of EIE at RMD Engineering college obtained her $\mathrm{PhD}$ (Electrical Engineering) from Anna University, Chennai in the year 2014. Her areas of interest include Electrical Engineering, Special Electrical machines, Artificial Intelligence, Biomedical Instrumentation, Programmable Logic Controllers. She has published 15 papers in reputed International Journals and Conference

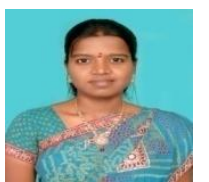

Mrs.N.Padmavathi is working as Assistant professor in the department of EIE at R.M.D Engineering College. Her areas of interest are Renewable Energy Systems, Converters in Solar Panels, soft computing. She has presented 05 papers in International Conferences and published Two papers in International journals.

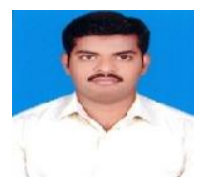

Mr.M.Aravindan obtained B.E. (EIE) Degree in 2005 from Shri Angalamman college of Engineering and Technology, Trichy and M.Tech. (Electronics and Instrumentation) Degree in 2012 from B.S Abdur Rahman University, Chennai. He is pursuing Ph.D under Anna University. He is in the teaching profession for the past 9 years and his area of interest is Process Control Instrumentation. He attended workshop in PID Control, Internet of Things (IoT) and Embedded systems. He is a Life member in ISOI and ISA. 